\title{
Explosive synchronization in weighted complex networks
}

\author{
I. Leyva, ${ }^{1,2}$ I. Sendiña-Nadal,,${ }^{1,2}$ J. A. Almendral, ${ }^{1,2}$ A. Navas, ${ }^{2}$ S. Olmi, ${ }^{3,4}$ and S. Boccaletti ${ }^{3}$ \\ ${ }^{1}$ Complex Systems Group, Univ. Rey Juan Carlos, 28933 Móstoles, Madrid, Spain \\ ${ }^{2}$ Center for Biomedical Technology, Univ. Politécnica de Madrid, 28223 Pozuelo de Alarcón, Madrid, Spain \\ ${ }^{3}$ CNR-Institute of Complex Systems, Via Madonna del Piano, 10, 50019 Sesto Fiorentino, Florence, Italy \\ ${ }^{4}$ INFN - Sezione di Firenze and CSDC, via Sansone 1, 50019 Sesto Fiorentino, Italy
}

(Received 25 July 2013; published 14 October 2013)

\begin{abstract}
The emergence of dynamical abrupt transitions in the macroscopic state of a system is currently a subject of the utmost interest. Given a set of phase oscillators networking with a generic wiring of connections and displaying a generic frequency distribution, we show how combining dynamical local information on frequency mismatches and global information on the graph topology suggests a judicious and yet practical weighting procedure which is able to induce and enhance explosive, irreversible, transitions to synchronization. We report extensive numerical and analytical evidence of the validity and scalability of such a procedure for different initial frequency distributions, for both homogeneous and heterogeneous networks, as well as for both linear and nonlinear weighting functions. We furthermore report on the possibility of parametrically controlling the width and extent of the hysteretic region of coexistence of the unsynchronized and synchronized states.
\end{abstract}

DOI: 10.1103/PhysRevE.88.042808

PACS number(s): 89.75.Hc, 89.75.Kd, 89.75.Da, 64.60.an

\section{INTRODUCTION}

One of the most significant challenges of present-day research is bringing to light the processes underlying the spontaneous organization of networked dynamical units. When a network passes from one to another collective phase under the action of a control parameter, the nature of the associated phase transition is disclosed by the behavior of the order parameter at criticality. In complex networks' theory [1,2] such phase transitions have been observed in the way a graph collectively organizes its architecture through percolation [3-5], and its dynamical state through synchronization, both for continuous $[6,7]$ and discrete [8] dynamical systems.

Abrupt transitions to synchronized states of networked phase oscillators were initially reported in a Kuramoto model [9] for a particular realization of a uniform frequency distribution (evenly spaced frequencies) and an all-to-all network topology [10]. Later on, the same finding was also described for both periodic [11] and chaotic [12] phase oscillators in the yet particular condition of a heterogeneous degree distribution with positive correlations between the node degree and the corresponding oscillator's natural frequency. Recently, Ref. [13] introduced a more general framework where explosive synchronization (ES) is obtained in weighted networks, where weights are selected to be proportional to the absolute value of the frequency of the oscillators in a way that produces positive correlations between the node input and the frequency of the oscillator.

The weighting procedure proposed in Ref. [13] inherently asymmetrizes each link of the network, favoring the interaction directions from lower to higher frequencies. In this work, we propose an alternative general framework for ES in complex networks, based on a weighting procedure which instead keeps the symmetric nature of the links. The method is inspired by our recent study of Ref. [14], where it is shown that ES can be obtained for any given frequency distribution, provided the connection network is constructed following a rule of frequency disassortativity; that is, that the synchronization clustering formation is prevented avoiding close frequencies to couple, in a network generation scheme ruled by dynamical properties, as the Achlioptas rule [4] works for the structural case in explosive percolation.

We here deal with the more general case of a network with given frequency distribution and architecture, and we show that a weighting procedure on the existing links, which combines information on the frequency mismatch of the two end oscillators of a link with that of the link betweenness, has the effect of inducing or enhancing ES phenomena for both homogeneous and heterogeneous graph topologies, as well as any symmetric or asymmetric frequency distribution. In addition, we show the general scaling properties of the obtained transition and provide analytical arguments in support of our claims.

\section{MODEL AND NUMERICAL RESULTS}

Without lack of generality, our reference is a network $\mathcal{G}$ of $N$ Kuramoto [9] phase oscillators, described by

$$
\frac{d \theta_{i}}{d t}=\omega_{i}+\frac{\sigma}{\langle k\rangle} \sum_{i=1}^{N} \Omega_{i j}^{\alpha} \sin \left(\theta_{j}-\theta_{i}\right),
$$

where $\theta_{i}$ is the phase of the $i$ th oscillator $(i=1, \ldots, N), \omega_{i}$ is its associated natural frequency drawn from a frequency distribution $g(\omega), \sigma$ is the coupling strength, $\langle k\rangle$ is the graph average connectivity $\left(\langle k\rangle \equiv \frac{2 L}{N}\right.$, with $L$ being the total number of links), and

$$
\Omega_{i j}^{\alpha}=a_{i j}\left|\omega_{i}-\omega_{j}\right|^{\alpha}
$$

is the weighted link for nodes $i, j$, with $a_{i j}$ being the elements of the adjacency matrix that uniquely defines $\mathcal{G}$ and $\alpha$ being a constant parameter which eventually modulates the weight. The strength of the $i$ th node (the sum of all its links weights) is then $s_{i}=\sum_{j} \Omega_{i j}^{\alpha}$. The classical order parameter for system (1) is $r(t)=\frac{1}{N}\left|\sum_{j=1}^{N} e^{i \theta_{j}(t)}\right|$, and the level of synchronization can be monitored by looking at the value of $R=\langle r(t)\rangle_{T}$, with $\langle\cdots\rangle_{T}$ denoting a time average over a conveniently large time span $T$. 
As the coupling strength $\sigma$ increases, system (1) undergoes a phase transition at a critical value $\sigma_{c}$ from the unsynchronized $(R \sim 1 / \sqrt{N})$ to the synchronous $(R=1)$ state, where all oscillators ultimately acquire the same frequency. In the following, we will describe the nature of such a transition as a function of the rescaled order parameter $\sigma /\langle k\rangle$. As for the stipulations followed in our simulations, the state of the network is monitored by gradually increasing $\sigma$ in steps $\delta \sigma=0.0005$, starting at $\sigma=0$. Whenever a step $\delta \sigma$ is made, a long transient (200 time units) is discarded before the data are recorded and processed. Moreover, as we are focusing on abrupt, irreversible transitions (and thus on expected associated hysteretic phenomena), we perform the simulations also in the reverse way, i.e., starting from a given value $\sigma_{\max }$ (where $R=1$ ), and gradually decreasing the coupling by $\delta \sigma$ at each step. In what follows, the two sets of numerical trials are termed forward and backward, respectively.

\section{A. Homogeneous networks}

We first report our results for the case of homogeneous graph topologies. For this purpose, we consider Erdös-Rényi (ER) random networks [15] of size $N$, and we describe how an explosive transition is induced for sufficiently large values of $\langle k\rangle$ and irrespectively on the specific frequency distribution $g(\omega)$. Figure 1(a) reports the results for $N=500$ and several frequency distributions $g(\omega)$ within the range $[0,1]$. For the simplest case of uniform frequency distribution $g(\omega)=1$,
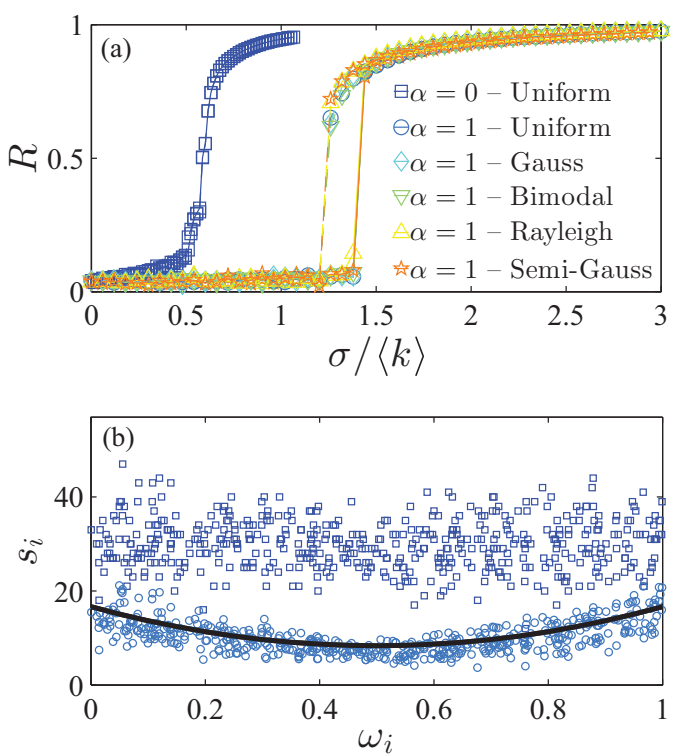

FIG. 1. (Color online) (a) Synchronization transitions for $N=$ 500 ER networks, $\langle k\rangle=30$, for unweighted case $(\alpha=0)$ (blue squares), and linearly weighted cases $(\alpha=1)$ with several frequency distributions within the range [0,1]: uniform, Gaussian, Gaussianderived, Rayleigh, and semi-Gaussian. Solid and dashed lines refer to the forward and backward simulations, respectively. (b) Node strengths $s_{i}$ (see text for definition) vs natural frequencies $\omega_{i}$, for the unweighted (dark blue squares) and weighted (light blue circles) networks reported in panel (a). Solid line is proportional to the analytical prediction $\left(\omega-\frac{a}{2}\right)^{2}+\frac{1}{4 a}$ in the thermodynamical limit of our model, with $a=1$ the width of the uniform frequency distribution (see text for more details). while the unweighted network $[\alpha=0$ in Eq. (2)] displays a smooth, second-order-like transition to synchronization [dark blue curve in Fig. 1(a)], the effect of a linear weighting $(\alpha=1)$ is that of inducing a sharp transition in the system, with an associated hysteresis in the forward (solid line) and backward (dashed line) simulations. This drastic change in the nature of the transition is independent of the frequency distribution $g(\omega)$, as long as they are defined in the same frequency range $[0,1]$ as shown in Fig. 1(a). The results are identical for symmetric distributions (homogeneous, Gaussian, a bimodal distribution derived from a Gaussian) and for asymmetric frequency distributions (Rayleigh, a Gaussian centered at 0 but just using the positive half). See details of the used frequency distributions in Ref. [16].

Figure 1(b) accounts for the existence of a parabolic relationship between the strengths and the natural frequencies of the oscillators associated with the passage from a smooth to an explosive phase transition. This relationship has been obtained analytically [see Eq.(7)] in the thermodynamical limit of the Kuramoto model and perfectly fits the numerical results shown as a solid line in Fig. 1(b). It has to be remarked that, while in Ref. [11] degree-frequency correlation features were imposed to determine explosiveness in the transition to synchronization, here the effect of the weighting is to let these correlation features between topology and dynamics spontaneously emerge, with the result of shaping a bipartitelike network where low- and high-frequency oscillators are the ones with maximal overall strength.

Further information about the nature and scaling properties of the transition induced by the linear weighting procedure is gained from Fig. 2, where it is shown the dependence of the scaled critical coupling $\sigma_{c} /\langle k\rangle$ on the average connectivity $\langle k\rangle$ and on the network size $N$. Precisely, Fig. 2(a) shows that, independently of $N$, a dynamical bifurcation exists at $\langle k\rangle \sim 17$, corresponding to the passage from a second- to a first-order-like phase transition. For the latter regime, the two branches expanding from $\langle k\rangle \gtrsim 17$ are associated with the hysteresis in the forward and backward simulations. The relative independence on $N$ can be explained considering that an important condition for ES to occur is that each node neighborhood must represent a statistically significant sample of the network frequencies up to give a close enough approximation to the global mean frequency, and therefore the synchronization frequency. To reach this target, the required sampling size $n$ for a given population size $N$ is usually calculated with the following formula [17]:

$$
n=\frac{N}{1+C^{2}(N-1)},
$$

where $C:=2 e / z_{\alpha / 2}, e$ is the error allowed, $1-\alpha$ is the confidence level, and $z_{\alpha / 2}$ is the upper $\alpha / 2$ percentage point of the standard normal distribution. Aside from the technical details, the important feature in the expression is that the sampling size converges to a finite value, even for an infinite population. This is exactly what Fig. 2(a) shows. Once the mean degree is large enough, each node has a neighborhood assuring that its neighbor frequency average is statistically accurate. Precisely, Fig. 2(a) suggests that $C \approx 0.24$, indicating that, for mean degrees greater than $\sim 17$, each node has a sufficiently large neighborhood independently 

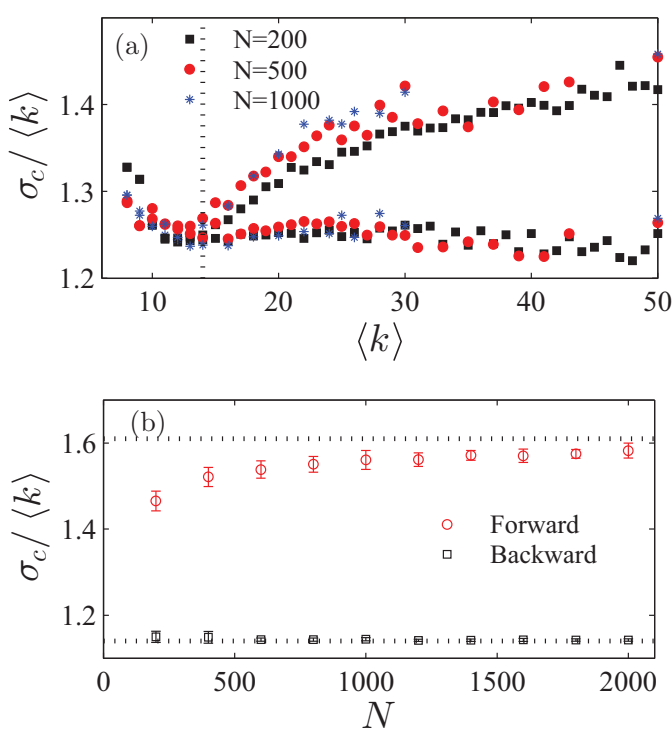

FIG. 2. (Color online) Critical scaled couplings $\sigma_{c} /\langle k\rangle$ at the onset of synchronization (forward simulation) and desynchronization (backward simulation) using a linear weighting procedure $\Omega_{i j}(\alpha=$ 1) as a function of (a) $\langle k\rangle$ for several ER network sizes $N$, and of (b) $N$ in all-to-all coupled networks. In panel (a) the vertical dashed line marks the passage from a smooth to an explosive phase transition. In both panels (a) and (b) the upper and lower branches correspond to forward and backward simulations, respectively. Each dot accounts for an average of at least 20 independent runs of uniform frequency distributions. Horizontal dashed lines in panel (b) are close to the analytical values defining the range of the hysteresis in the thermodynamical limit for the Kuramoto model (see explanation in text). Frequencies are uniformly distributed in the range $[0,1]$.

of the population size $N$. Figure 2(b) shows how the scaled critical couplings defining the hysteresis of the ES transition converge to constant values for the Kuramoto model (all-to-all coupling) when $N$ increases which are quite close to those obtained in the thermodynamical limit of the Kuramoto model discussed in the analytical section.

Furthermore, the weighting procedure inducing ES is quite general, as a large family of detuning dependent functions can be used. As an example, Fig. 3 describes the case of nonlinear weighting procedures; that is, $\alpha \neq 1$ in Eq. (2). There, we set again $N=500$ and $\langle k\rangle=30$ and consider both ER graphs [Fig. 3(a)] and a regular random network [Fig. 3(b)], i.e., a network where each node has exactly the same number of connections $\left(k_{i}=\langle k\rangle=30\right)$ with the rest of the graph. This latter case has been obtained by a simple configuration model [18], imposing a $\delta$-Dirac degree distribution. The results in Fig. 3 show that the generic nonlinear function of the frequency mismatch given by Eq. (2) is able to induce ES in both topologies, and that the effect of a superlinear $(\alpha>1)$ weighting [sublinear $(\alpha<1)$ weighting] is that of enhancing (reducing) the width of the hysteretic region.

\section{B. Heterogeneous networks}

So far, we have considered only homogeneous degree distributions. In order to properly describe the passage from a homogeneous to a heterogeneous degree distribution, we rely
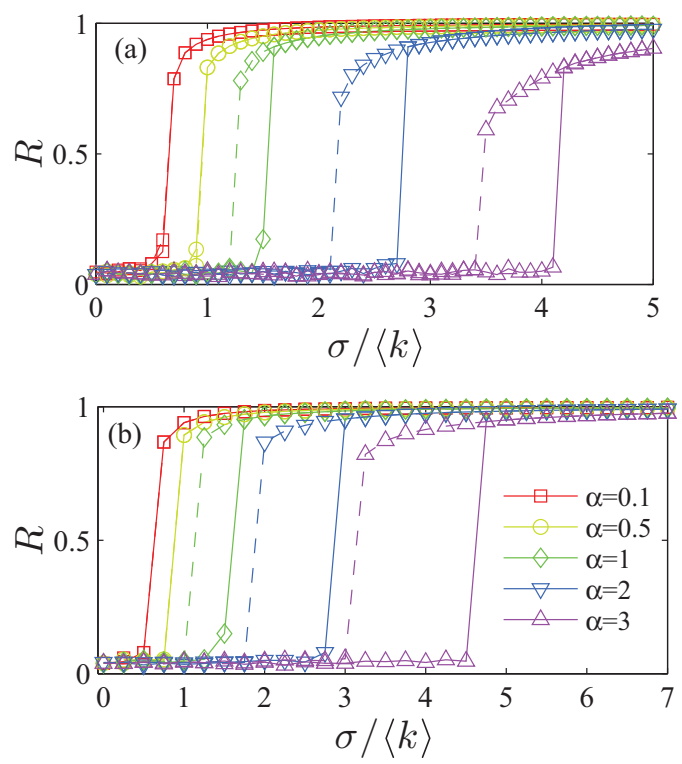

FIG. 3. (Color online) Synchronization transitions for ER networks, $N=500$, uniformly distributed frequencies in the [0,1] range, and nonlinear weighting functions $\Omega_{i j}^{\alpha}$. Both plots consider several $\alpha$ values, from sublinear to superlinear weighting [see legend in panel (b)]. (a) ER networks, $\langle k\rangle=30$, (b) regular random networks, $k=30$. In all cases, forward and backward simulations correspond respectively to solid and dashed lines.

on the procedure introduced in Ref. [19]. Such a technique, indeed, allows constructing graphs with the same average connectivity $\langle k\rangle$ and grants one the option of continuously interpolating from ER to scale-free (SF) networks [20], by tuning a single parameter $0 \leqslant p \leqslant 1$. With this method, networks are grown from an initial small clique by sequentially adding nodes up to the desired graph size. Each newly added node has a probability $p$ of forming random connections with already existing vertices, and a probability $1-p$ of following a preferential attachment rule [20] for the selection of its connections. As a result, the limit $p=1$ induces an ER configuration, whereas the limit $p=0$ corresponds to a SF network with degree distribution $P(k) \sim k^{-3}$.

Let us set $N=1000$ and $\langle k\rangle=30$ and, after the network construction, let us randomly distribute the oscillators' frequencies in the interval $[0,1]$ and use again a linear weighting function $\Omega_{i j}=a_{i j}\left|\omega_{i}-\omega_{j}\right|$. The comparative results are reported in Fig. 4 for different networks ranging from a completely regular random network to networks with increasing degree of heterogeneity (check the degree distributions in the inset of Fig. 4) and it is easy to see that heterogeneity in the degree distribution actually opposes the onset of explosive synchronization. Actually, the latter is only suppressed when the second moment of the degree distribution diverges (SF). A similar qualitative scenario (not shown) is obtained also for different frequency distributions, network sizes, and (superlinear or sublinear) weighting functions, allowing one to conclude that heterogeneous degree distributions require a different weighting approach, where the information on frequency mismatch has to be properly combined with local or global information on the network topology. 


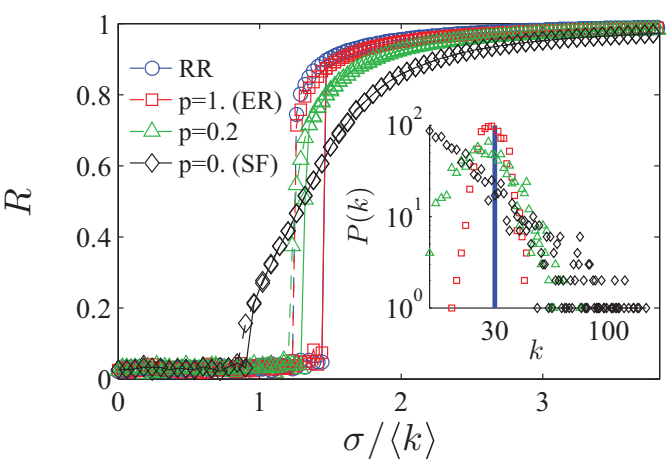

FIG. 4. (Color online) Explosive synchronization vs degree of heterogeneity. Synchronization transitions as a function of the coupling strength for linearly weighted networks $(\alpha=1)$ with the same average connectivity $\langle k\rangle=30$ and different networks: a regular random (RR) network with homogeneous degree (blue circles), and networks constructed using the model referenced in the text, $p=1$ (ER, red squares), $p=0.2$ (green triangles), and $p=0$ (SF, black diamonds). In all cases, forward and backward simulations correspond respectively to solid and dashed lines. Inset shows log-log plot of the four corresponding degree distributions.

The problem closely resembles what was called, in past years, the paradox of heterogeneity [21] where increasing the heterogeneity in the connectivity distribution of an unweighted network led to an overall deterioration of synchrony, despite the associated reduction of the network's shortest path. That paradox was lately solved by proving optimal synchronization conditions when proper weighting procedures are implemented on the graph's links accounting for either local [22] or global [23] information on the specific network topology. Therefore, in analogy with what was reported in Ref. [23], we consider a new weighting function

$$
\widetilde{\Omega}_{i j}=a_{i j}\left|\omega_{i}-\omega_{j}\right| \frac{\ell_{i j}^{\beta}}{\sum_{j \in \mathcal{N}_{i}} \ell_{i j}^{\beta}},
$$

with $\beta$ being a parameter and $\ell_{i j}$ being the edge betweenness associated with the link $a_{i j}$ [24], defined as the number of shortest paths between pairs of nodes in the network that run through that edge.

The results are reported in Fig. 5(a). While the case $\beta=0$ (black triangles, already shown in Fig. 4) corresponds to a smooth transition, the effect for $\beta \neq 0$ in Eq. (3) is highly nontrivial. Precisely, moderate (positive or negative) values of $\beta$ establish in system (1) an abrupt transition to synchronization. However, increasing $\beta$ beyond a critical value leads system (1) to display again a smooth and reversible character of the transition.

In its turn, Fig. 5(b) reports the hysteresis' area [the area of the plane $(R, \sigma /\langle k\rangle)$ covered by the hysteretic region] as a function of $\beta$, obtained by an ensemble average over 10 different forward and backward simulations of system (1) together with the weighting function (3), each one starting from a different realization of the uniform frequency distribution. The plot reveals the existence of an optimal condition around $\beta=0.5$ where the width of the hysteresis is maximized. Therefore, $\beta$ can be seen as an operational parameter through which one can control and regulate the width and extent in $\sigma$ of the hysteresis
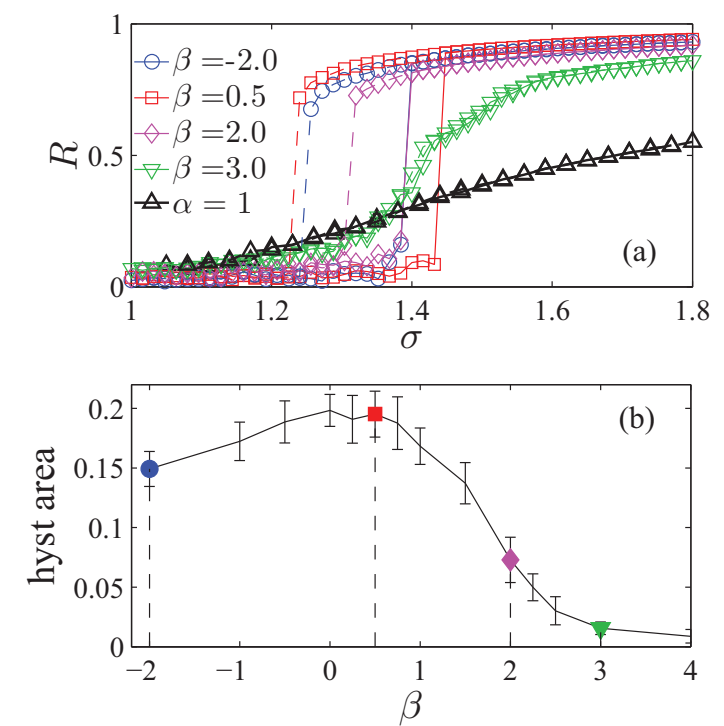

FIG. 5. (Color online) (a) Synchronization transitions for SF networks using different schemes of coupling weighting. In black triangles, the link between nodes $i$ and $j$ is weighted using $\Omega_{i j}^{\alpha}$ with $\alpha=1$ (as in Fig. 4), while the rest of cases refer to the weighting function $\widetilde{\Omega}_{i j}$ of Eq. (3), with the values of the $\beta$ parameter given in the legend. In all cases, forward and backward simulations correspond, respectively, to continuous and dashed lines. (b) Area of the hysteretic region vs $\beta$. Each point is an average of 10 different simulations, each one starting from a different realization of the frequency distributions. In all cases, $\langle k\rangle=30, N=1000$, and natural frequencies are uniformly distributed in the interval $[0,1]$.

associated with the irreversible nature of ES. The latter can be of interest for controlling the range of coupling strength for which system (1) can be used to originate magnetic-like states of synchronization, i.e., situations in which an originally unsynchronized configuration, once entrained to a given phase by an external pacemaker acting for a limited time lapse, is able to permanently stay in a synchronized configuration [14].

\section{ANALYTICAL RESULTS}

In order to study the onset and nature of the explosive transition, we must analytically examine the behavior of the system in the thermodynamic limit. Let us consider the paradigmatic case in which $N$ oscillators form a fully connected graph, as the original Kuramoto model, but with weights $\Omega_{i j}=\left|\omega_{i}-\omega_{j}\right|$. Then, the dynamical equations are

$$
\dot{\theta}_{i}=\omega_{i}+\frac{\sigma}{N} \sum_{j=1}^{N} \Omega_{i j} \sin \left(\theta_{j}-\theta_{i}\right),
$$

for $i=1, \ldots, N$.

By considering the following definitions:

$$
\begin{gathered}
\frac{1}{N} \sum_{j=1}^{N} \Omega_{i j} \sin \theta_{j}:=A_{i} \sin \phi_{i}, \\
\frac{1}{N} \sum_{j=1}^{N} \Omega_{i j} \cos \theta_{j}:=A_{i} \cos \phi_{i},
\end{gathered}
$$


the dynamical equations are usually expressed [25] in terms of trigonometric functions as

$$
\dot{\theta}_{i}=\omega_{i}+\sigma A_{i} \sin \left(\phi_{i}-\theta_{i}\right) .
$$

While these transformations are the same as those used in the original Kuramoto model, now there is an explicit dependence on $i$ in the quantities $A_{i}$ and $\phi_{i}$. In order to continue our analysis, we will then assume some mild approximations.

In the corotating frame, the phases must verify $\omega_{i}=$ $\sigma A_{i} \sin \left(\theta_{i}-\phi_{i}\right)$ to have a static solution (i.e., $\dot{\theta}_{i}=0$ ), which in the thermodynamic limit reads

$$
\omega=\sigma A_{\omega} \sin \left(\theta_{\omega}-\phi_{\omega}\right) .
$$

The definition of $A_{\omega}$ and $\phi_{\omega}$ implies that

$$
F(\omega):=A_{\omega} \sin \phi_{\omega}=\int g(x)|w-x| \sin \theta(x) d x,
$$

whose second derivative verifies

$$
F^{\prime \prime}(\omega)=\int g(x) 2 \delta(w-x) \sin \theta(x) d x=2 g(\omega) \sin \theta(\omega),
$$

using the distributional derivative of the signum function. Likewise, if we consider

$$
G(\omega):=A_{\omega} \cos \phi_{\omega}=\int g(x)|w-x| \cos \theta(x) d x,
$$

its second derivative verifies

$$
G^{\prime \prime}(\omega)=2 g(\omega) \cos \theta(\omega)
$$

Then, Eq. (4) takes the form

$$
\frac{2}{\sigma} g(\omega) \omega=F^{\prime \prime}(\omega) G(\omega)-F(\omega) G^{\prime \prime}(\omega) .
$$

Let us work out $F(\omega)$ and $G(\omega)$. When all oscillators are close to synchronization, we can assume that $\cos \theta(x) \approx R$, thus

$$
G(\omega) \approx R \int g(x)|w-x| d x=R s(\omega),
$$

where $s(\omega)$ is just the strength of a node with intrinsic frequency $\omega$. Therefore, Eq. (5) can be approximated by

$$
\frac{2}{R \sigma} g(\omega) \omega=F^{\prime \prime}(\omega) s(\omega)-F(\omega) s^{\prime \prime}(\omega),
$$

which is a second-order ordinary differential equation (ODE) whose integration yields $F(\omega)$. Notice that when $s(\omega)$ is a rather involved function, Eq. (6) is already an approximation, and we can just consider a polynomial expansion in $\omega$ to obtain an analytical expression of $F(\omega)$.

For instance, given a uniform distribution $g(\omega)$ in the interval $[-a / 2,+a / 2]$, the resulting strength is a secondorder polynomial,

$$
s(\omega)=a\left[\left(\frac{\omega}{a}\right)^{2}+\frac{1}{4}\right],
$$

which perfectly fits our numerical simulations [see Fig. 1(b)], even though it has been deduced for a complete graph. Then, the integration of Eq. (6) results in

$$
F(\omega)=a \frac{\left[1+4\left(\frac{\omega}{a}\right)^{2}\right] \arctan \left(\frac{2 w}{a}\right)-(2+\pi) \frac{w}{a}}{(4+\pi) \sigma R},
$$

using the initial condition $F(0)=0$, since $g(\omega)$ is a symmetric function [thus $F(\omega)$ is an odd function], and the consistency equation

$$
F(\omega)=\int g(x)|\omega-x| \sin \theta(x) d x=\int \frac{|\omega-x|}{2} F^{\prime \prime}(x) d x .
$$

Therefore, since $F^{\prime \prime}(\omega)=2 g(\omega) \sin \theta(\omega)$, we find that

$$
\sin \theta(\omega)=\frac{1}{\sigma R} H\left(\frac{2 \omega}{a}\right),
$$

where

$$
H(z):=\frac{4}{4+\pi}\left[\frac{z}{1+z^{2}}+\arctan (z)\right] .
$$

To determine how the order parameter $R$ depends on the coupling constant $\sigma$, we use

$$
R=\int g(x) \cos \theta(x) d x=\int g(x) \sqrt{1-\sin ^{2} \theta(x)} d x,
$$

which is an implicit equation in $R$. When $\sigma R \geqslant \frac{2+\pi}{4+\pi} \approx 0.72$, $\sin \theta(x) \leqslant 1$ for all $x$, which means that all oscillators are frequency locked, so

$$
R=\int_{-\frac{a}{2}}^{\frac{a}{2}} g(x) \sqrt{1-\left[\frac{1}{\sigma R} H\left(\frac{2 x}{a}\right)\right]^{2}} d x .
$$

When $\sigma R \leqslant \frac{2+\pi}{4+\pi}$, only those oscillators with frequency in the interval $\left[-\omega^{*}, \omega^{*}\right]$ are locked, where

$$
\omega^{*}:=\frac{a}{2} H^{-1}(\sigma R),
$$

thus

$$
R=\int_{-\frac{a}{2} H^{-1}(\sigma R)}^{\frac{a}{2} H^{-1}(\sigma R)} g(x) \sqrt{1-\left[\frac{1}{\sigma R} H\left(\frac{2 x}{a}\right)\right]^{2}} d x .
$$

Hence, if we define

$$
\ell(\mu):= \begin{cases}1 & \text { if } \quad \mu \geqslant \frac{2+\pi}{4+\pi} \\ H^{-1}(\mu) & \text { if } \quad 0 \leqslant \mu<\frac{2+\pi}{4+\pi}\end{cases}
$$

and

$$
I(\mu):=\int_{0}^{\ell(\mu)} \sqrt{1-\left[\frac{1}{\mu} H(z)\right]^{2}} d z,
$$

Eq. (8) takes the form

$$
\frac{\mu}{\sigma}=I(\mu)
$$

where $\mu=\sigma R$. Therefore, given a coupling constant $\sigma$, the value of $R$ is computed by solving this implicit equation in $\mu$. Notice that, geometrically, the solutions are the points where the straight line passing through the origin with slope $1 / \sigma$ intersects $I(\mu)$.

The main feature characterizing $I(\mu)$ is its inflection point at $\frac{2+\pi}{4+\pi}$, at which the curve changes from being concave up to concave down [see Fig. (6)]. This implies that, depending 

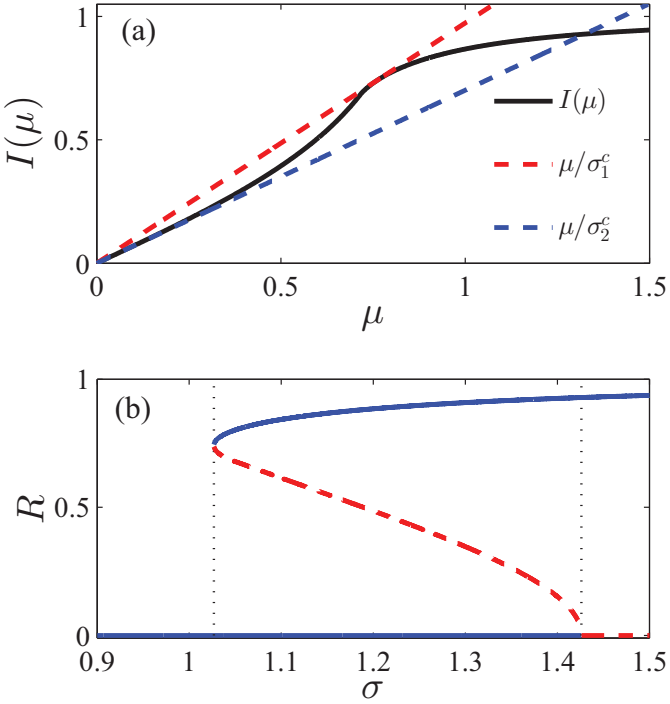

FIG. 6. (Color online) (a) $I$ as a function of $\mu=\sigma R$ (solid curve) as given by Eq. (9). The dashed lines are those straight lines whose intersection with $I$ marks the backward $\left(\sigma_{1}^{c}\right)$ and forward $\left(\sigma_{2}^{c}\right)$ critical points of ES for an all-to-all connected network and for a uniform frequency distribution. (b) The corresponding synchronization order parameter $R$ as a function of the coupling strength. Solid (dashed) curves correspond to the stable (unstable) solution. Dotted vertical lines mark the region of hysteresis defined by $\sigma_{1}^{c}$ and $\sigma_{2}^{c}$ in panel (a).

on $\sigma$, there are three qualitatively different type of solutions. When $\sigma$ is small, we have the trivial solution $R=0$ since the straight line and $I(\mu)$ only intersect at $\mu=0$. This situation changes when $\sigma$ is such that the slope of the straight line is tangent to $I(\mu)$ [i.e., when $\sigma=1.03$, corresponding to the red dashed line in Fig. 6(a)]. When $\sigma$ is greater than this value, we enter into the region where the hysteresis takes place since, now, there are three values of $R$, two of them are stable solutions $(R=0$ and $R \approx 1)$ and the third one is a unstable solution [see Fig. 6(b)]. The solution $R \approx 1$ appears therefore abruptly, due to the existence of the inflection point. This behavior changes when the slope of the straight line is tangent to $I(0)$ [i.e., when $\sigma=1.43$, corresponding to the blue dashed line in Fig. 6(a)], which is the point where the stable solution
$R=0$ collapses with the unstable one, becoming unstable [see Fig. 6(b)]. Notice that the numerical values obtained for the Kuramoto model for large $N$ in Fig. 2(b) are quite close to those predicted by the theory.

\section{CONCLUSIONS}

In conclusion, we have introduced a weighting procedure based on the link frequency mismatch and on the link betweenness to induce an explosive transition to synchronization in a generic complex network of phase oscillators and for a generic distribution of the frequencies. As a consequence of this procedure, topological and dynamical correlation features spontaneously emerge, with the result of shaping a bipartitelike network where frequency disassortativity prevails.

In this scenario, the passage from a smooth to an abrupt transition has found to be fully rescalable, and critically depends only on the average connectivity and not on the network size.

In addition, we analytically proved that our weighting procedure yields a first-order-like transition whose hysteresis extent is calculated. Moreover, the theoretical framework allows for a geometrical interpretation of the explosive transition in which the weighting imposes a multivalued Kuramoto phase order parameter, in contrast with the classical model.

The present results could provide significant insights into the study of real complex networks such as power grids which can be modeled as networks of phase oscillators whose coupling may depend on the dynamics of the nodes [26].

\section{ACKNOWLEDGMENTS}

The authors acknowledge Alessandro Torcini for many fruitful discussion on the subject and the computational resources and assistance provided by CRESCO, the center of ENEA in Portici, Italy. Financial support from the Spanish Ministerio de Ciencia e Innovación (Spain) under projects FIS2011-25167, FIS2009-07072, and of Comunidad de Madrid (Spain) under project MODELICO-CM S2009ESP1691, are also acknowledged. S. O. acknowledges the MIUR project CRISISLAB PNR 2011-2013.
[1] S. Boccaletti, V. Latora, Y. Moreno, M. Chavez, and D. U. Hwang, Phys. Rep. 424, 175 (2006).

[2] S. N. Dorogovtsev, A. V. Goltsev, and J. F. F. Mendes, Rev. Mod. Phys. 80, 1275 (2008).

[3] R. Cohen, D. ben-Avraham, and S. Havlin, Phys. Rev. E 66, 036113 (2002); M. Karsai, J-Ch. Anglès d'Auriac, and F. Iglói, ibid. 76, 041107 (2007); G. Li, L. A. Braunstein, S. V. Buldyrev, S. Havlin, and H. E. Stanley, ibid. 75, 045103 (2007).

[4] D. Achlioptas, R. M. D’Souza, and J. Spencer, Science 323, 1453 (2009).

[5] Y. S. Cho, J. S. Kim, J. Park, B. Kahng, and D. Kim, Phys. Rev. Lett. 103, 135702 (2009); F. Radicchi and S. Fortunato, ibid. 103, 168701 (2009); P. Grassberger, C. Christensen, G. Bizhani, S.-W. Son, and M. Paczuski, ibid. 106, 225701 (2011).
[6] S. Boccaletti, J. Kurths, G. Osipov, D. L. Valladares, and C. S. Zhou, Phys. Rep. 366, 1 (2002).

[7] A. Arenas, A. Díaz-Guilera, J. Kurths, Y. Moreno, and C. S. Zhou, Phys. Rep. 469, 93 (2008).

[8] P. G. Lind, A. Nunes, and J. A. C. Gallas, Physica A 371, 100 (2006).

[9] Y. Kuramoto, Chemical Oscillations, Waves and Turbulence (Springer, Berlin, 1984).

[10] D. Pazó, Phys. Rev. E 72, 046211 (2005).

[11] J. Gómez-Gardeñes, S. Gómez, A. Arenas, and Y. Moreno, Phys. Rev. Lett. 106, 128701 (2011).

[12] I. Leyva, R. Sevilla-Escoboza, J. M. Buldú, I. Sendiña-Nadal, J. Gómez-Gardeñes, A. Arenas, Y. Moreno, S. Gómez, R. JaimesReátegui, and S. Boccaletti, Phys. Rev. Lett. 108, 168702 (2012). 
[13] X. Zhang, X. Hu, J. Kurths, and Z. Liu, Phys. Rev. E 88, 010802(R) (2013).

[14] I. Leyva, A. Navas, I. Sendiña-Nadal, J. A. Almendral, J. M. Buldú, M. Zanin, D. Papo, and S. Boccaletti, Sci. Rep. 3, 1281 (2013).

[15] P. Erdós and A. Rényi, Publ. Math. Debrecen 6, 290 (1959).

[16] The details of the used distributions are: (i) Gaussian $g(\omega)=$ $(1 / a \sqrt{2 \pi}) e^{-\frac{(\omega-0.5)^{2}}{2 a^{2}}}$ with $a=0.23$, (ii) a bimodal distribution derived from a Gaussian $g(\omega)=(1 / a \sqrt{2 \pi}) e^{-\frac{\omega^{2}}{2 a^{2}}}$ if $\omega<0.5$, and $g(\omega)=(1 / a \sqrt{2 \pi}) e^{-\frac{(\omega-1.0)^{2}}{2 a^{2}}}$, (iii) Rayleigh distribution $g(\omega)=$ $\left(\omega / b^{2}\right) e^{-\frac{\omega^{2}}{2 b^{2}}}$ with $b=10$ and normalized to the $[0,1]$ range, $(i v)$ distribution derived from a Gaussian centered at 0 but just using the positive half $g(\omega)=(1 / a \sqrt{2 \pi}) e^{-\frac{(\omega-0.5)^{2}}{2 a^{2}}}$ with $\omega>0$, named as semi-Gaussian in Fig. 1(a).

[17] G. W. Cochran, Sampling Techniques (John Willey \& Sons, New York, 1977), pp 74-76.
[18] E. A. Bender and E. R. Canfield, J. Combin. Theory Ser. A 24, 296 (1978).

[19] J. Gómez-Gardeñes and Y. Moreno, Phys. Rev. E 73, 056124 (2006).

[20] A.-L. Barabási and R. Albert, Science 286, 509 (1999).

[21] T. Nishikawa, A. E. Motter, Y.-C. Lai, and F. C. Hoppensteadt, Phys. Rev. Lett. 91, 014101 (2003).

[22] A. E. Motter, C. S. Zhou, and J. Kurths, Europhys. Lett. 69, 334 (2005); Phys. Rev. E 71, 016116 (2005).

[23] M. Chavez, D.-U. Hwang, A. Amann, H. G. E. Hentschel, and S. Boccaletti, Phys. Rev. Lett. 94, 218701 (2005).

[24] M. E. J. Newman and M. Girvan, Phys. Rev. E 69, 026113 (2004).

[25] S. H. Strogatz, Phys. D 143, 1 (2000).

[26] A. E. Motter, S. A. Myers, M. Anghel, and T. Nishikawa, Nat. Phys. 9, 191 (2013). 